\title{
An Automatic Method for Optimizing Venturi Shape in Cavitation Flows
}

\author{
Vijay Shankar' ${ }^{1}$ Anton Lundberg ${ }^{2}$, Kristian Frenander ${ }^{2}$, Lars Landström², Taraka Pamidi' \\ Örjan Johansson ${ }^{1}$ \\ ${ }^{1}$ Luleå University of Technology \\ Luleå, Sweden \\ vijay.shankar@1tu.se \\ ${ }^{2}$ Chalmers University of Technology \\ Gothenburg, Sweden
}

\begin{abstract}
In order to lower the energy consumption of the fibrillation stage for the pulp and paper industry, a new technology need to be innovated and developed. The current research work deals with a new innovative concept based on creating cavitation in the pulp flow. A venturi nozzle is designed and optimized, where hydrodynamic cavitation is achieved by the so called Venturi effect. This paper focuses on the development of an automatic method for venturi shape optimization. The process of cavitation is hard to control and can cause high mechanical wear, therefore an optimization study of the venturi shape is performed with two main objectives. Firstly, to achieve cavitation that is sustained for as long as possible downstream and secondly to avoid cavitation at the walls. The developed method is a type of two-level optimization based on neural networks and evolutionary optimization. A number of simulations are executed and the optimization is then performed on a solver approximation instead of the real solver, which considerably reduces computation time. The obtained results show the optimal venturi configuration and the relative importance of each shape parameter. The optimal configuration is a clear improvement of the baseline configuration and an improvement also compared to all of the tested samples, thereby validating the optimization method.
\end{abstract}

Keywords: Cavitation, Neural networks, Optimization, Pulp \& Paper, Venturi nozzle

\section{Introduction}

The pulp and paper industry consumes about $52 \%$ of the energy consumed in the Swedish manufacturing sector on a yearly basis [1]. This makes it the individual sector with the largest energy consumption in Sweden's industry and a very relevant sector on which to focus research efforts on energy efficiency. It is also interesting to reduce the energy consumption within the paper mills, as their production of energy from the biological residue of the pulping process is quite significant. In 2012 the energy production from bio-fuels in the Swedish pulp and paper industry amounted to 42.3 TWh [2]. If the energy consumption within the sector could be further reduced the paper mills could eventually become net exporters of green bio-energy and thus contribute to a climate-neutral society. Papers should clearly describe the background of the subject, the authors work, including the methods used, results and concluding discussion on the importance of the work. Papers are to be prepared in English and SI units must be used. Technical terms should be explained unless they may be considered to be known to the conference community.

\subsection{Disc Refiners}

When one observes the production line, noting where energy is consumed, the disc refiners, where the wood chips are grinded into fibres, stand out as a particularly energy demanding process step. With current techniques energy consumption can be up to several tens of megawatts for each disc refiner [3]. These refiners process the fibres mechanically with two or more rotating discs. The rotating discs are patterned with slots and bars that at the same time force the fibres from the centre of the refiner to the periphery and grind the chips as they pass over them. Research indicate that the passing of the slots over the fibres give rise to cavitation [4], which contribute to the fibrillation of the fibres [5]. Cavitation is however undesirable despite its positive properties, as it also gives rise to significant mechanical wear and fatigue on the refiner plates. 


\subsection{Proposed Future Design}

An entirely novel technology for fibre refining has been suggested in [6] with the purpose of significantly lowering the energy consumption in this step of the process. Their idea is based around cavitation, but instead of viewing it as an undesirable by-product that causes wear and fatigue on the machinery, they propose that the power of the bubble collapse should be utilized for the fibrillation and processing of the pulp fibres.

The design should induce hydrodynamic cavitation by running the pulp stream through a venturi nozzle. The cavitation bubbles thus induced will then collapse when the pipe expands and the static pressure recovers, and the idea is to focus the bubble collapse on the surface of the fibres to achieve fibrillation and processing. This means that the venturi nozzle needs to be carefully designed so that the cavitation can be controlled and used to concentrate the processing energy to the fibres and provide effective refinement of the fibres in the flow.

\section{Optimization Method}

An efficient method for shape optimization was recently developed by Lundberg et al. [7]. This method was originally developed for vehicle shape optimization but has in this project been adapted and extended for venturi shape optimization. A short summary of the method is presented below. For details on method choices etc. the reader is referred to [7]. The method is a type of two-level optimization, meaning that the optimization is performed on a solver approximation instead of the real solver, which considerably reduces computation time. A schematic of the method can be seen in Fig. 1.

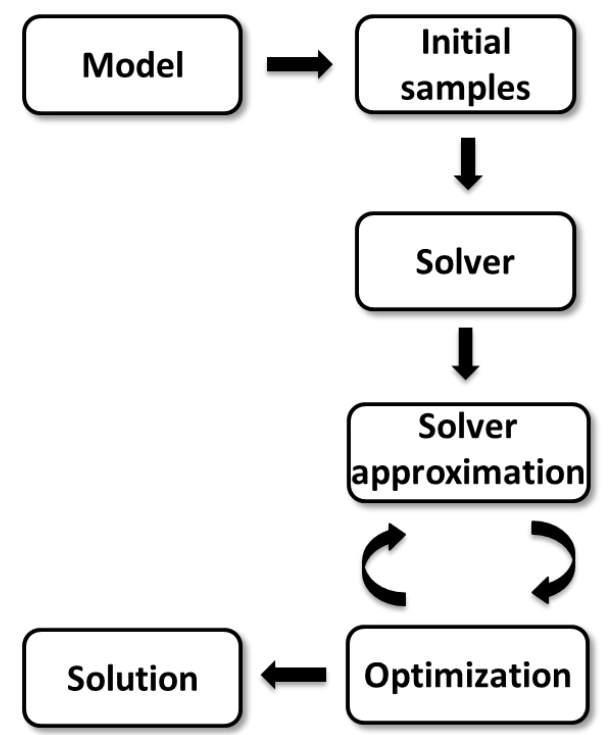

Fig. 1: Schematic of two-level shape optimization method from [7].

A number of venturi shape configurations are generated from one initial model using mesh morphing. The configurations are chosen using Latin hypercube sampling in order to achieve a statistically reliable representation of the configuration space. A database is created from CFD simulations on these configurations. The database is used to train a neural network which acts as an approximation to the simulations. This means that instead of running a CFD simulation to obtain results for each configuration tested in the optimization algorithm, approximative values are obtained from the neural network. This is considerably faster and allows the optimization algorithm to include a large number of configurations. Finally, an optimal venturi shape is determined using multi-objective particle swarm optimization.

\subsection{Mesh Morphing}

An initial venturi model is created and meshed in ANSA. A new mesh is created for each configuration by updating a number of design parameters in the initial model using mesh morphing. After morphing the mesh is regenerated. The mesh morphing allows for precise updates of the geometry and by defining a morphing case this process is fully automated. 


\subsection{Multi-Objective Particle Swarm Optimization}

A multi-objective particle swarm optimization is used to determine the optimal configuration from the solver approximation. This is a fast-evolutionary method that has been found to perform well compared to other widely used evolutionary methods, such as the non-dominated sorting genetic algorithm (NSGA-II) [8]. In [7] it was found that the best performance was achieved with 100 particles and 200 generations. Initial tests showed that 200 generations was more than enough to find a converged solution. These values were therefore also used in the current investigation.

\section{Modelling and Measuring Cavitation}

Cavitation in a liquid can occur when the static pressure in the liquid drops below the vapour pressure. Hydrodynamic cavitation is cavitation that arises from pressure variations as a result of velocity changes in the liquid, typically created by driving the fluid through a narrowing geometry, for example a venturi nozzle. As the liquid passes through the venturi the flow speed increases and the static pressure reduces as both mass and energy must be conserved in the passage through the nozzle. Hydrodynamic cavitation can therefore be induced at otherwise ambient conditions and represent the most energy efficient way of inducing cavitation [5].

\subsection{Solver Settings}

The flow was solved in Fluent using a pressure-based Navier-Stokes solver with the k- $\omega$ SST turbulence model and the energy equation included. The multiphase calculations utilized a mixture model, which is a simplified Euler-Euler model. Cavitation was modelled with the Schnerr and Sauer model [9] assuming an abundant presence of nuclei for bubble formation. The vapour pressure was modelled as temperature dependent by means of a piecewise-linear function, with the vapour pressure at the inlet temperature $345 \mathrm{~K}$ set to $33.7 \mathrm{kPa}$.

Multiphase calculations, especially cavitation flows, are inherently unstable and hard to get to converge meaning that the way the case was run needed to be fine-tuned. This was implemented by turning on the different models gradually, so that the case was allowed to converge to a reasonable level for every model implemented in that stage. The basic process is explained in Fig. 2. After reading the mesh, the flow was initialised and a number of iterations were performed using firstorder discretization schemes before switching to second-order discretization. The flow was allowed to converge before activating the multiphase and cavitation models. Output started when the flow had stabilized.

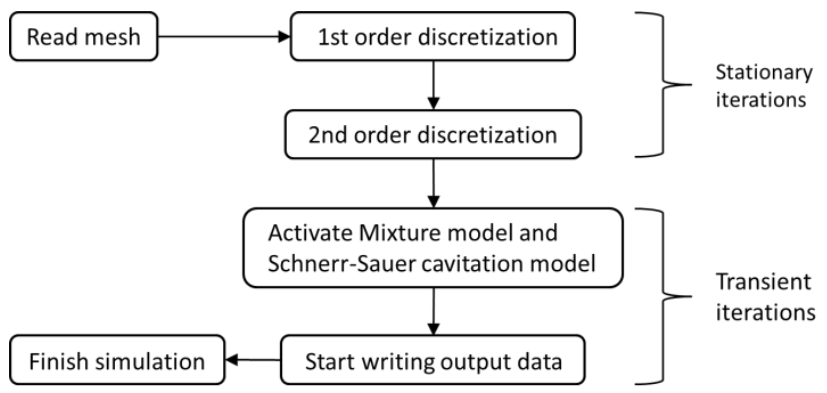

Fig. 2: Flowchart showing the solver process.

\subsection{Objective Function}

Vital to the optimization process is the objective function, i.e. measuring the goal of the optimization. The objective function for the venturi optimization is not entirely straightforward. There are primarily two problems; how to measure cavitation and how to sample an unsteady, stochastic process. The flow upstream of the venturi consists of one single phase, liquid water. The venturi creates cavitation bubbles where the water has changed phase to vapour. That is, the cavitation introduces a second phase - water vapour. The volume fraction of vapour can thus be used as a measure of the amount of cavitation in the flow. The cavitation process is unsteady and there are fluctuations in the flow. The vapour fraction was therefore averaged over a period of time larger than the time scale of the fluctuations.

There were two main objectives for the venturi: to achieve a cavitation that is sustained for as long as possible and to avoid cavitation at the walls. These objectives were implemented by creating two zones, one in the centre of the flow downstream from the venturi and one near the wall, and measuring the average vapour fractions in these. The two 
objectives are shown in Fig. 3 and Fig.4. The near wall zone was defined as a thin band along the entire channel wall. The centre zone radius was half the channel radius.

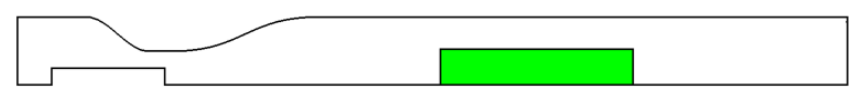

Fig. 3: Optimization objective 1: maximize vapour fraction in centre zone (filled area).

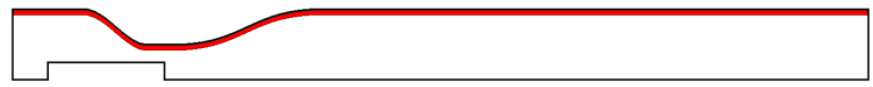

Fig. 4: Optimization objective 2: minimize vapour fraction in near-wall zone (filled area).

\section{Venturi Shape Optimization}

The aim of the venturi shape optimization was to obtain a design where cavitation was induced and sustained in the flow. It was also desired that the cavitation bubbles remain in the centre of the flow as a mean of reducing stress and wear for the mechanical structure. In order to achieve these objectives an optimization study was performed with three parameters. Simulations were performed on a flow consisting of pure water and the study did not consider the effect fibres might have on cavitation.

\subsection{Initial Venturi Design}

Previous experiments and simulations have found that cavitation tends to attach to the geometry walls, see for instance [10]. This is because it mainly forms where the liquid structure is broken up, which typically occurs near solid objects. To counter this effect and obtain a cavitation flow centred in the geometry an obstruction was added to the venturi. The concept can be seen as a development of the ideas put forward in [11]. The modified venturi design is shown in Fig. 5. The problem is axisymmetric and simulations were performed on a $2 \mathrm{D}$ model. A detail of this computational model is shown in Fig. 6.

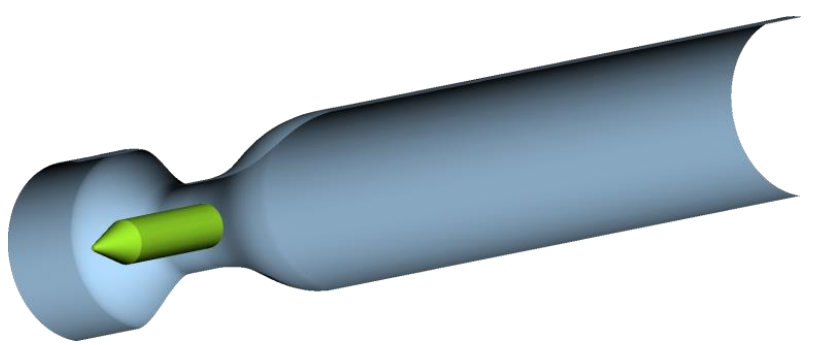

Fig. 5: 3D cut of initial venturi design with flow obstruction visible.

Table 1: Venturi Shape Configuration and Respective Objective Values. Obstruction Radius and Position and Reported Relative to the Throat Radius, RT.

\begin{tabular}{|c|c|c|c|c|c|}
\hline Configuration & $r_{o}$ & $x_{o}$ & $P_{\text {in }}$ & Vapour fraction, & Vapour fraction, \\
\hline & {$[-]$} & {$[-]$} & {$[\mathrm{bar}]$} & centre [\%] & wall [\%] \\
\hline Minimum & $0.30 \mathrm{R}_{\mathrm{t}}$ & $-0.20 \mathrm{R}_{\mathrm{t}}$ & 3.0 & - & - \\
\hline Maximum & $0.70 \mathrm{R}_{\mathrm{t}}$ & $0.40 \mathrm{R}_{\mathrm{t}}$ & 5.0 & - & - \\
\hline Baseline & $0.50 \mathrm{R}_{\mathrm{t}}$ & $0.00 \mathrm{R}_{\mathrm{t}}$ & 3.00 & 47.6 & 6.7 \\
\hline 1 & $0.62 \mathrm{R}_{\mathrm{t}}$ & $-0.15 \mathrm{R}_{\mathrm{t}}$ & 3.66 & 28.2 & 7.5 \\
\hline 2 & $0.49 \mathrm{R}_{\mathrm{t}}$ & $0.07 \mathrm{R}_{\mathrm{t}}$ & 3.87 & 94.2 & 31.8 \\
\hline 3 & $0.38 \mathrm{R}_{\mathrm{t}}$ & $0.31 \mathrm{R}_{\mathrm{t}}$ & 4.14 & 9.9 & 53.2 \\
\hline 4 & $0.50 \mathrm{R}_{\mathrm{t}}$ & $-0.07 \mathrm{R}_{\mathrm{t}}$ & 3.22 & 42.3 & 4.8 \\
\hline 5 & $0.59 \mathrm{R}_{\mathrm{t}}$ & $0.20 \mathrm{R}_{\mathrm{t}}$ & 3.42 & 38.0 & 1.7 \\
\hline
\end{tabular}




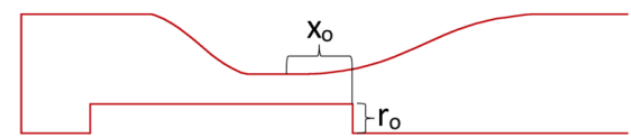

Fig. 6: Detail of the venturi in computational 2D model showing the geometrical design parameters.

\subsection{D Study: Three Parameters}

Three design parameters were studied for the venturi: obstruction radius $\left(r_{o}\right)$, obstruction position $\left(x_{o}\right)$ and inlet pressure $\left(P_{i n}\right)$. The obstruction position was measured as the position of the end of the obstruction relative to the centre of the throat. The geometrical parameters are shown in Fig. 6 . These parameters were allowed to vary between the values shown in the top of Table I.

A configuration study was performed with 9 initial samples. The number of samples were chosen according to [7] as $2^{3}=8$, which together with a baseline configuration makes a total of 9 configurations. The geometry for each configuration was created automatically from the baseline configuration using mesh morphing in ANSA. A simulation of each configuration was run in Fluent after which the objective function was evaluated with a post-processing script in Matlab. The configurations and their respective objective values are shown in Table I.

\subsection{Optimal Venturi Shape}

A neural network was trained with the simulation data in Table I to act as a solver approximation. An optimal venturi shape was determined from this solver approximation using multi-objective particle swarm optimization. Since the optimization had two objectives there is not one single global optimum but rather a collection of optimal points called a Pareto front, see Fig. 7. Each of these points corresponds to a specific configuration with approximative vapour fraction values given by the neural network. It was regarded more important to avoid cavitation at the wall than to maximize the centre zone vapour fraction. An optimal configuration was therefore chosen as the point on the Pareto front with the lowest wall vapour fraction, that is, the bottom left point in Fig. 7 A CFD simulation was performed on the optimal configuration to determine the actual vapour fraction values. The optimal configuration and a comparison of the predicted and simulation vapour fractions are shown in Table II.

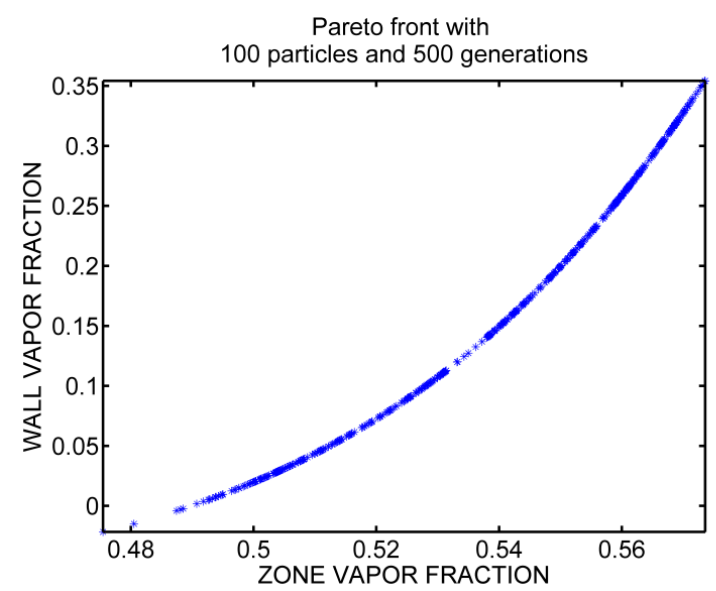

Fig. 7: Predicted optimal combinations (Pareto front) of center zone vapour fraction and wall vapour fraction for the venturi.

\subsection{Accuracy}

As can be seen in Table II the optimization method did not offer a good prediction of the objectives, with the predicted centre zone vapour fraction almost three times larger than the actual simulation value. It did however produce a good solution. The optimization was aimed at avoiding cavitation at the wall, which is precisely what was achieved. From Table I it can be seen that the lowest wall vapour fraction of the tested configurations is $1.7 \%$. The optimal configuration has a wall vapour fraction of $0.8 \%$, less than half that. Compared to the wall vapour fraction of the baseline configuration at $6.7 \%$, the optimal configuration is a clear improvement.

In [7] it was found that the optimization accuracy mainly depends on two factors: solver accuracy and database size. There is an apparent trade-off between accuracy and cost here. Increasing both solver accuracy and database size will lead 
to an increased simulation time and/or simulation power, which were the limiting factors of this study. There are a lot of factors governing the solver accuracy, but the main factor for this study was the inherit instability of cavitation processes. The ideal solution is to simulate a large number of time steps and average over these. However, this is seldom possible due to constraints on simulation power and time. The strategy applied in this project was to look at the fluctuations in cavitation and average over a time larger than these fluctuations.

A more accurate prediction would likely have been achieved with a larger database and longer simulations. This would however have been more time-consuming and required more computational resources. Despite the small database of only 9 CFD simulations, the optimal solution had the lowest wall vapour fraction out of all tested configurations and was a clear improvement from the baseline configuration. So even though the uncertainty of the objective values were high and only a small number of configurations were tested, the optimization method managed to improve the venturi design. The improved design was the best tested, which means that the optimization method is more efficient than a trivial approach of simply testing several configurations and choosing the best one. However, the method is most suited for initial parameter studies with large design changes, without a need for accurate predictions.

Table 2: Optimal Configuration. Predicted and Simulation Values. Obstruction Radius and Position are Reported Relative to the Throat Radius, RT.

\begin{tabular}{|c|c|c|c|c|c|}
\hline \multicolumn{2}{|c|}{} & \multicolumn{2}{c|}{ Parameter } & \multicolumn{2}{c|}{ Vapour fraction } \\
\hline & $r_{O}$ & $x_{O}$ & $P_{\text {in }}$ & Centre & Wall \\
\hline $\begin{array}{c}\text { Predicted } \\
\text { Simulation }\end{array}$ & $0.62 \mathrm{R}_{\mathrm{t}}$ & $-0.19 \mathrm{R}_{\mathrm{t}}$ & $3.00 \mathrm{bar}$ & $47.5 \%$ & $-2.2 \%$ \\
\cline { 2 - 6 } & $0.62 \mathrm{R}_{\mathrm{t}}$ & $-0.19 \mathrm{R}_{\mathrm{t}}$ & $3.00 \mathrm{bar}$ & $17.3 \%$ & $0.8 \%$ \\
\hline
\end{tabular}

\subsection{Parameter Impact}

A large number of design configurations were sampled randomly over the configuration space and their respective objectives were approximated using the neural network. By computing the correlation of all these configurations to the objectives a qualitative comparison of each parameter's impact can be made. The correlation coefficient matrix $C$ is defined as [12].

$$
C(i, j)=\frac{\operatorname{cov}(i, j)}{\sqrt{\operatorname{cov}(i, i) \operatorname{cov}(j, j)}}
$$

where $\operatorname{cov}(i, j)$ is the covariance matrix. From this the correlation coefficient for each parameter to the two objectives was extracted. A positive correlation means that an increase of that parameter will lead to an increase of the objective as well. Similarly, a negative correlation means that an increase of that parameter will lead to a decrease of the objective. The magnitude is a measure of the strength of the correlation.

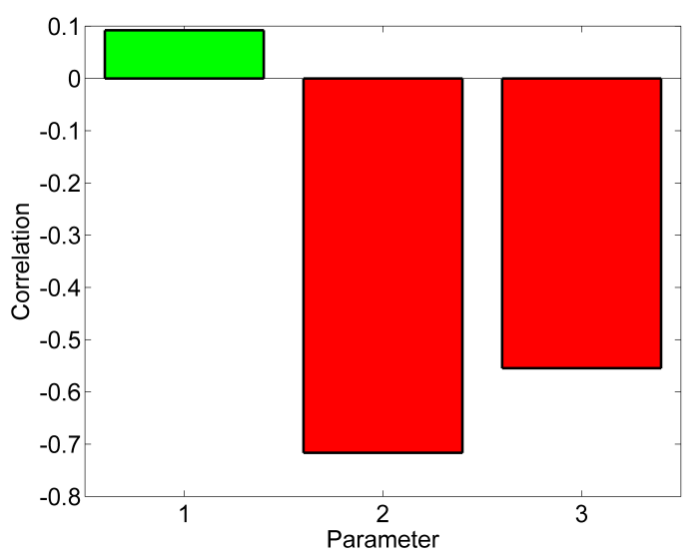

Fig. 8: Correlation of obstruction radius (1), obstruction position (2) and inlet pressure (3) to centre zone vapour fraction. 
The correlation coefficient of each parameter to the centre zone vapour fraction is shown in Fig. 8. It shows that there is a small positive correlation between obstruction radius and centre zone vapour fraction and a large negative correlation for obstruction position and inlet pressure. This means that an increase in obstruction radius will likely lead to an increased centre zone vapour fraction, whereas the inverse relation exists for the two other parameters. Judging from the relative magnitudes of the correlation, the important parameters are obstruction position and inlet pressure. The effect of obstruction radius is very small compared to the effects of these.

The case is clearer cut for wall vapour fraction, see Fig. 9. The important parameters are obstruction radius and inlet pressure, with obstruction position having almost no effect at all. Increasing obstruction radius will likely lead to a decreased wall vapour fraction, whereas the opposite is true for inlet pressure.

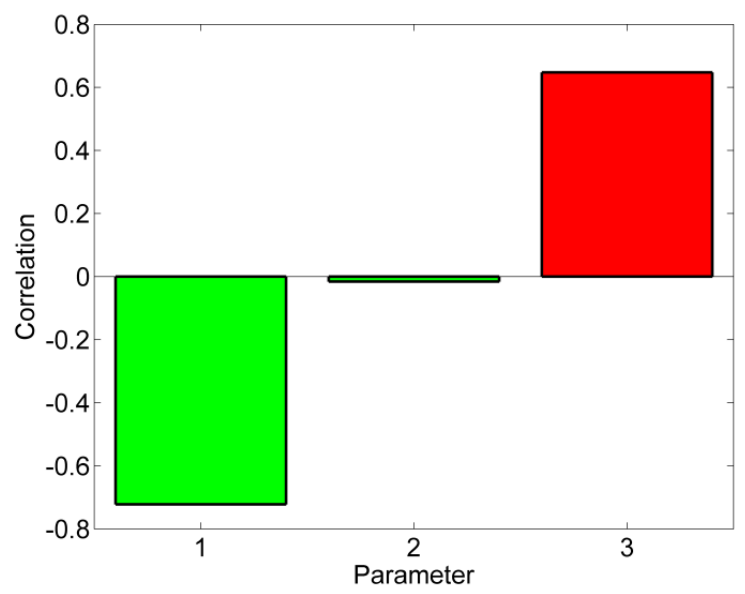

Fig. 9: Correlation of obstruction radius (1), obstruction position (2) and inlet pressure (3) to wall vapour fraction.

\section{Conclusion}

An automatic method for optimizing venturi shape in cavitation flows has been developed. The method was applied in a parameter study of a venturi with an obstruction, with the aim of achieving and sustaining cavitation in the centre of the flow and avoiding cavitation at the wall. Three parameters were studied: obstruction radius, obstruction position and inlet pressure. Despite a small number of tested configurations and large fluctuations in the solution, the optimization method managed to find a solution that was an improvement of the baseline configuration. The optimal configuration was an improvement also compared to all of the tested samples, meaning that the optimization method performs well.

The parameter study showed that obstruction position and inlet pressure were important for cavitation in the centre flow, while obstruction radius and inlet pressure were important for cavitation at the wall.

\section{Future Work}

As stated earlier, the optimization method is very promising but there is room for further improvements. The focus of such studies should be to improve the prediction accuracy and stability. One way to do this could be to refine the solver procedure to reach a better accuracy and to improve the optimization objectives. The optimal shape is currently being studied in 3D simulations. An interesting extension of this work would be to also account for the effects of fibres in the flow by performing 3D simulations with fibre modelling.

\section{Acknowledgements}

The project has been carried out at $\AA$ F in collaboration with ÅForsk, Energimyndigheten (Swedish Energy Agency), Luleå University of Technology, Holmen, SCA and Stora Enso.

\section{References}

[1] Swedish Energy Agency, "ET2013:22 Energiläget 2013 (Government report)," 2013.

[2] Statistics Sweden, "Industrins årliga energianvändning 2012 (Government report)," 2014. 
[3] M. Illikainen, E. Härkönen, M. Ullmar and J. Niinimäki, "Power consumption distribution in a TMP refiner: comparison of the first and second stages," TAPPI Journal, vol. 6, no. 9, pp. 18-23, 2007.

[4] O. Eriksen and L. Hammar, "Refining mechanisms and development of TMP properties in a low-consistency refiner (Technical report),” Trondheim: Paper and Fibre Research Institute (PFI), 2005.

[5] P. R. Gogate and A. B. Pandit, "Hydrodynamic cavitation reactors: a state of the art review," Reviews in Chemical Engineering, vol. 17, no. 1, pp. 1-85, 2001.

[6] Ö. Johansson and L.-O. Landström, "Slutrapport: Hur kan resonansfenomen utnyttjas för att minska energiförbrukningen vid framställning av papper (Technical report)," Stockholm: ÅForsk, 2010.

[7] A. Lundberg, P. Hamlin, D. Shankar, A. Broniewicz, T. Walker, C. Landström, "Automated aerodynamic vehicle shape optimization using neural networks and evolutionary optimization," SAE Int. J. Passeng. Cars - Mech.Syst., vol. 8, no. $1,2015$.

[8] C. A. C. Coello, G. T. Pulido and M. S. Lechuga, "Handling multiple objectives with particle swarm optimization," IEEE Transactions on Evolutionary Computation, vol. 8, no. 3, pp. 256-279, 2004.

[9] G. Schnerr and J. Sauer, "Physical and numerical modeling of unsteady cavitation dynamics," Fourth International Conference on Multiphase Flow, New Orleans, USA, vol. 1, 2001.

[10] S. Barre, J. Rolland, G. Boitel, E. Goncalves and R.F. Patella, "Experiments and modeling of cavitating flows in venturi: attached sheet cavitation," European Journal of Mechanics-B/Fluids, vol. 28, no. 3, pp. 444-464, 2009.

[11] T. A. Bashir, A. G. Soni, A. V. Mahulkar and A. B. Pandit, "The cfd driven optimisation of a modified venturi for cavitational activity," The Canadian Journal of Chemical Engineering, vol. 89, no. 6, pp. 1366-1375, 2011.

[12] M. H. Beale, M. T. Hagan and H. B. Demuth, "Matlab neural network toolbox:User's guide (manual)," 2014. 